\title{
Top food sources of percentage of energy, nutrients to limit and total gram amount consumed among US adolescents: National Health and Nutrition Examination Survey 2011-2014
}

\author{
Ana Carolina Leme ${ }^{1,2}$, Tom Baranowski ${ }^{2}$, Debbe Thompson ${ }^{2}$, Sonia Philippi ${ }^{1}$, \\ Carol $\mathrm{O}^{\prime} \mathrm{Neil}^{3}$, Victor Fulgoni $1 \mathrm{Il}^{4}$ and Theresa Nicklas ${ }^{2, *}$ \\ ${ }^{1}$ School of Public Health, University of São Paulo, São Paulo, Brazil: ${ }^{2}$ USDA/ARS Children's Nutrition Research \\ Center, Baylor College of Medicine, 1100 Bates Avenue, Houston, TX 77030, USA: ${ }^{3}$ School of Nutrition and Food \\ Science, Louisiana State University Agricultural Center, Baton Rouge, LA, USA: ${ }^{4}$ Nutrition Impact, LLC, Battle Creek, \\ MI, USA
}

Submitted 16 February 2018: Final revision received 18 June 2018: Accepted 24 September 2018: First published online 23 November 2018

\begin{abstract}
Objective: To identify most commonly consumed foods by adolescents contributing to percentage of total energy, added sugars, SFA, Na and total gram intake per day.

Design: Data from the National Health and Nutrition Examination Survey (NHANES) 2011-2014.

Setting: NHANES is a cross-sectional study nationally representative of the US population.

Participants: One $24 \mathrm{~h}$ dietary recall was used to assess dietary intake of 3156 adolescents aged 10-19 years. What We Eat in America food category classification system was used for all foods consumed. Food sources of energy, added sugars, SFA, Na and total gram amount consumed were sample-weighted and ranked based on percentage contribution to intake of total amount.

Results: Three-highest ranked food subgroup sources of total energy consumed were: sugar-sweetened beverages (SSB; 7.8\%); sweet bakery products (6.9\%); mixed dishes - pizza (6.6\%). Highest ranked food sources of total gram amount consumed were: plain water $(33 \cdot 1 \%)$; SSB $(15 \cdot 8 \%)$; milk (7.2\%). Three highest ranked food sources of total Na were: mixed dishes - pizza ( $8.7 \%)$; mixed dishes Mexican (6.7\%); cured meats/poultry (6.6\%). Three highest ranked food sources of SFA were: mixed dishes - pizza (9.1\%); sweet bakery products ( $8 \cdot 3 \%)$; mixed dishes - Mexican (7.9\%). Three highest ranked food sources of added sugars were: SSB (42.1\%); sweet bakery products (12.1\%); coffee and tea (7.6\%).

Conclusions: Identifying current food sources of percentage energy, nutrients to limit and total gram amount consumed among US adolescents is critical for designing strategies to help them meet nutrient recommendations within energy needs.
\end{abstract}

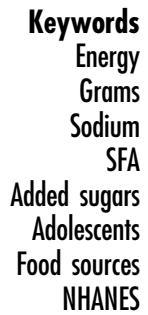

Energy intake in excess of energy expenditure and higher intakes of added sugars, SFA and $\mathrm{Na}$ contribute to unhealthy weight gain and/or chronic non-communicable diseases, including CHD and type 2 diabetes ${ }^{(1-3)}$. Adolescence is an important period to understand dietary intake, because it is a period of transition from childhood to adulthood when teens become more independent in their food choices ${ }^{(4)}$. Given the overabundance of foods and beverages available, targeting reductions in the amounts and percentage contribution of the most commonly consumed foods with these nutrients among adolescents may be an effective strategy to more closely align their diets with current dietary recommendations ${ }^{(5)}$. This approach may have the positive effect of increasing intakes of foods and beverages that contain the food groups to encourage and of decreasing nutrients to limit in the diets of adolescents ${ }^{(5-9)}$.

Cross-sectional data from six nationally representative surveys of food intake (1989 to 2010) with US children and adolescents revealed that total energy intake increased considerably from 1989 to 2004, and subsequently declined through $2010^{(10)}$. Seven food sources were 
consistently major contributors to total energy intake across all time points: sugar-sweetened beverages (SSB), pizza, higher-fat milk, grain-based desserts, breads, pasta dishes and savoury snacks. Intakes of higher-fat milk, meats and processed meat products, ready-to-eat cereals, hamburgers, fried potatoes, $100 \%$ juice and vegetables decreased from 1989 to 2010, while intakes of low-fat milk, poultry, sweet snacks, candies, and tortillas and corn-based dishes increased over this period ${ }^{(10)}$. Although there may have been a decrease in total energy intake, adolescents were still consuming foods high in energy, which might affect overall diet quality ${ }^{(11-13)}$. Food sources of energy, added sugars and SFA make major contributions to intakes of nutrients of public health concern (i.e. dietary fibre, vitamin $\mathrm{D}, \mathrm{Ca}$ and $\mathrm{K}$ ) as well as other essential nutrients ${ }^{(11-14)}$.

SFA and $\mathrm{Na}$ are considered overconsumed nutrients that may pose a public health concern due to cardiovascular and metabolic diseases ${ }^{(5)}$. In addition, the 2015 Dietary Guidelines for Americans recommend limiting intakes of energy from solid fat (i.e. SFA and trans-fatty acids) and added sugars $^{(15)}$. Linear trends in intakes of solid fats and added sugars decreased in adolescents aged 2-18 years from 1994 to 2010, but the mean intakes continue to exceed recommended limits ${ }^{(13)}$. The top food sources of SFA were: cheese; milk; frankfurters, sausages, luncheon meats; beef; other fats and oils (e.g. butter and animal fats, margarine, cream cheese); milk desserts; cake, cookies, quick breads, pastry pies; and crackers, popcorn, pretzels, chips $^{(1)}$. The US population is consuming $\mathrm{Na}$ in excess of dietary guideline levels and snacking behaviours are contributing to the excessive intake over the 30 years, especially among adolescents. Salty snacks and desserts and sweets (i.e. grain-based desserts) were the top food sources of $\mathrm{Na}^{(16)}$. Average daily $\mathrm{Na}$ intake was highest among older (14-18 years old) school-aged children (3565 (SE 120) $\mathrm{mg}$ ) and lowest among females (2919 (sE 74) mg) compared with younger (6-10 years old) children (3051 (SE 61) mg) and males (3584 (SE 102) mg), all exceeding recommended amounts ${ }^{(12)}$.

Understanding current food sources of energy intake and nutrients to limit among adolescents in the USA is important for designing age-specific strategies to help meet current dietary recommendations ${ }^{(5)}$. Although detailed lists of food sources of energy and nutrients among US children ( $2-18$ years) and adults ( $\geq 19$ years) from the National Health and Nutrition Examination Survey (NHANES) 2003-2006 have been published ${ }^{(1,17)}$, no study using recent NHANES data has examined the top food sources consumed by adolescents aged 10-19 years. The present study identifies the most commonly consumed food sources by adolescents (10-19 years) in terms of their contribution to energy, nutrients to limit and the total gram amount of foods consumed using nationally representative data from NHANES 2011-2014.

\section{Methods}

\section{NHANES overview}

The NHANES is a cross-sectional survey that uses a multistage stratified area probability sample of noninstitutionalized individuals, that provides nationally representative estimates of the US population ${ }^{(18)}$. An Overview of NHANES ${ }^{(18)}$ including the purpose, plan and operations, sample design, weighting procedures, analytic guidelines $^{(19)}$ and response rates and population totals ${ }^{(20)}$ is available elsewhere. In addition, a recent review article has been published describing NHANES in more detail, including analytical considerations and uses to inform public policy ${ }^{(21)}$. The survey examines approximately 5000 persons each year and data are released in 2-year cycles. Data were collected via a mobile examination centre throughout the year on both weekends and weekdays $^{(22)}$.

\section{Study sample}

The WHO defines adolescents as those people between 10 and 19 years of age ${ }^{(23)}$. Therefore, data from adolescents aged 10-19 years participating in the NHANES 20112012 and 2013-2014 were combined to increase sample size, resulting in a final analytic sample of 3156 after excluding those with unreliable records as defined by US Department of Agriculture (USDA) staff. The unweighted response rates for the adolescents aged 10-19 years for NHANES 2011-2012 and 2013-2014 were 75.8 and 78.5\%, respectively $^{(24,25)}$. Demographic information ${ }^{(26)}$ and selfreported physical activity levels ${ }^{(27)}$ were determined from the NHANES survey. Physical activity was classified into three levels: sedentary, moderate and vigorous, according to the individuals' intensity level in a typical week ${ }^{(27)}$. The NHANES employs protocols and procedures that ensure confidentiality and protect individual participants from identification $^{(26,27)}$.

\section{Determination of dietary intake data}

Dietary intake data were obtained from the in-person $24 \mathrm{~h}$ dietary recall interview using an automated multiplepass method ${ }^{(28)}$. The source of food composition data was based on the 2011-2012 and 2013-2014 Food Patterns Equivalents Databases, which convert the foods and beverages in the Food and Nutrient Database for Dietary Studies to USDA Food Patterns components ${ }^{(29)}$. Added sugars were defined by the USDA as all sugars used as ingredients in processed and prepared foods such as breads, cakes, soft drinks, jams, chocolates and ice cream, or eaten separately or added to foods at the table (MyPlate). Added sugars were determined using the NHANES cycle appropriate MyPyramid Equivalents Databases for USDA Survey Food Codes from 2001 to 2008 (versions 1.0 and 2.0) until these were replaced by the Food Patterns Equivalents Database in 2005 for each 
NHANES release (Food Patterns). Trained interviewers collected the recall data in English or Spanish, as appropriate. Participants aged 12 years or older completed the dietary interview on their own, but proxyassisted interviews were conducted with participants aged 10 and 11 years. Detailed description of the dietary interview methods is provided in the NHANES Dietary Interview Procedure Manual ${ }^{(30)}$. A single $24 \mathrm{~h}$ dietary recall administered in a sufficiently large population sample can adequately provide data to estimate population mean intakes ${ }^{(31)}$.

\section{Food groupings}

The What We Eat In America (WWEIA) food category classification system $^{(32)}$ was used to classify all foods consumed (obtained from the individual food file and combination foods were evaluated at the item level of each food in the combination). From fifteen main groups of the 2011-2012 and 2013-2014 WWEIA food categorization system ${ }^{(32)}$, only twelve were included in the present study: Milk and Dairy; Protein Foods; Mixed Dishes; Grains; Snacks and Sweets; Fruits; Vegetables; Beverages, Non-alcoholic; Fats and Oils; Condiments and Sauces; Sugars; and Water. The other food groups were excluded because they were not commonly consumed in this target population (e.g. Infant Formula and Baby Food; Other (e.g. protein and nutritional powders); Alcoholic Beverages). From these twelve main groups, forty-one food subgroups (e.g. quick breads and bread products, readyto-eat cereals, flavoured milk, yoghurt, cheese) were included to determine the rank order of contributors to total energy, $\mathrm{Na}$, SFA, added sugars and total gram amount of foods/beverages consumed ${ }^{(32)}$. The food categories in the online supplementary material, Supplemental Table 1, describe the assignment of the 137 specific WWEIA food categories to major food groups ( $n$ 12) and subgroups $(n 41)^{(5)}$.

\section{Anthropometric measures}

Height and weight were measured using procedures outlined in the NHANES Anthropometry Procedures Manual $^{(33)}$. BMI was calculated as body weight (in kilograms) divided by the square of height (in metres) ${ }^{(34)}$. The Centers for Disease Control and Prevention's growth chart programs were used to determine BMI-for-age percentiles; children with BMI $\geq 85$ th and $<95$ th percentile and BMI $\geq 95$ th percentile were considered overweight or obese, respectively ${ }^{(35)}$.

\section{Statistical analysis}

Analyses were conducted using the statistical software packages SAS version 9.2 and SUDAAN version 11 . Appropriate weights were used to adjust for oversampling of certain groups, non-response by some sample persons and the complex sample design of NHANES to obtain nationally representative estimates. Descriptive statistics (means and percentages, with their standard errors) for food sources on a population level using SAS PROC RATIO were determined for energy, Na, SFA, added sugars and total gram amount consumed reported on the day of the $24 \mathrm{~h}$ dietary recall. Mean per capita energy, total grams, Na, SFA and added sugars consumed from each food group were expressed as a percentage of the total to allow relativity across sex and age groups.

\section{Results}

\section{Demographics and lifestyle characteristics}

The sample from NHANES 2011-2014 included 3156 respondents aged 10-19 years with 53\% of the adolescents aged $10-14$ years, who were equally represented by sex. Mean BMI was 23.3 (SE 0.2 ) kg/m², with 63.3 (SE 1.6) \% normal weight, 16.2 (SE 0.9)\% overweight and 20.5 (SE $1.4) \%$ obese. Most adolescents reported being nonHispanic White $(54.5$ ( $\operatorname{se~3.3)\% )~and~head~of~household~}$ education status being more than high school (56.8 (SE 2.4)\%). Most adolescents reported vigorous physical activity status (59.6 (SE 1.51)\%) followed by moderate (28.3 ( $\operatorname{se~} 1.4) \%$ ). On the day of the $24 \mathrm{~h}$ dietary recall, the mean energy intake was 8774 (SE 95) kJ (2097 (SE 22.7) kcal; Table 1).

Table 1 Demographic and lifestyle characteristics of US adolescents aged $10-19$ years ( $n$ 3156), National Health and Nutrition Examination Survey 2011-2014

\begin{tabular}{lrr}
\hline Age group & $n$ & $\%$ \\
\hline Total & 3156 & $100 \cdot 0$ \\
Sex & & \\
$\quad$ Female & 1579 & $50 \cdot 2$ \\
$\quad$ Male & 1577 & $49 \cdot 8$ \\
Weight status & & \\
$\quad$ Normal weight & 1924 & $63 \cdot 3$ \\
Overweight & 546 & $16 \cdot 2$ \\
Obese & 656 & $20 \cdot 5$ \\
Race/ethnicity & & \\
$\quad$ Hispanic & 665 & $15 \cdot 1$ \\
Non-Hispanic White and other & 784 & $54 \cdot 5$ \\
Non-Hispanic Black & 863 & $14 \cdot 6$ \\
$\quad$ Non-Hispanic non-Black Asian & 844 & $15 \cdot 9$ \\
Head of household education & & \\
$\quad$ Low (less than high school) & 806 & $21 \cdot 6$ \\
Mid (high school) & 700 & $21 \cdot 6$ \\
High (more than high school) & 1560 & $56 \cdot 8$ \\
Physical activity & & \\
$\quad$ Sedentary & 406 & $12 \cdot 1$ \\
Moderate & 845 & $28 \cdot 3$ \\
Vigorous & 1873 & $59 \cdot 6$ \\
& & \\
$\quad$ BMl (kg/m ${ }^{2}$ ) & Mean & $\mathrm{SE}$ \\
\cline { 2 - 3 } Alcohol intake (g) & $23 \cdot 3$ & $0 \cdot 2$ \\
Poverty index ratio & $0 \cdot 9$ & $0 \cdot 2$ \\
Total energy intake (kJ) & $2 \cdot 3$ & $0 \cdot 1$ \\
Total energy intake (kcal) & 8774 & 95 \\
\hline & 2097 & $22 \cdot 7$ \\
\hline
\end{tabular}




\section{Contribution of foods to total energy intake}

Table 2 shows the dietary sources of energy consumed from the WWEIA's food subgroups. The more specific food categories are presented in the online supplementary material, Supplemental Table 2. The ten highest ranked subgroups contributed $55 \cdot 1 \%$ of total energy intake, including: $7 \cdot 8 \%$ from SSB ( $4.4 \%$ from soft drinks); $6.9 \%$ from sweetened bakery products $(2.8 \%$ from cookies and brownies); $6 \cdot 6 \%$ from mixed dishes - pizza; $5.8 \%$ from bread, rolls and tortillas $3.0 \%$ from yeast bread); $5 \cdot 3 \%$ from mixed dishes - grain-based $(2 \cdot 3 \%$ from pasta mixed dishes, excludes macaroni and cheese); $5 \cdot 1 \%$ from mixed dishes - Mexican (3.2\% from burritos and tacos); $4.6 \%$ from savoury snacks ( $2 \cdot 0 \%$ from tortilla, corn, other chips); $4.6 \%$ from mixed dishes - sandwiches (1.2\% from hamburgers and $1.1 \%$ from chicken/turkey sandwiches); $4.4 \%$ from milk (2.4\% from milk, reduced fat); and $4.0 \%$ from poultry $(2.2 \%$ from chicken, whole pieces).

\section{Contribution of foods to total grams consumed}

All the top beverage sources ( $n$ 9) contributed $67 \cdot 2 \%$ of total grams consumed. Plain water contributed $33 \cdot 1 \%$ with tap water the most consumed category (19.5\%). The other three highest ranked beverage subgroups (and categories) were: $15 \cdot 8 \%$ from SSB ( $8.6 \%$ from soft drinks); $7 \cdot 2 \%$ from milk (3.9\% from milk, reduced-fat); and $4.9 \%$ from coffee and tea $(3 \cdot 8 \%$ from tea). Further, all the top food sources ( $n$ 32) contributed $31.5 \%$ of total grams consumed and the five highest ranked food subgroups (and categories) were: $2.9 \%$ from fruits $(0.8 \%$ from apples); $2.7 \%$ from mixed dishes - grain-based ( $1.4 \%$ from pasta mixed dishes, excludes macaroni and cheese); $1.9 \%$ from mixed dishes pizza; $1.7 \%$ from mixed dishes - Mexican (1.2\% from

Table 2 Food sources* of mean and percentage of energy (kcal)† intake among US adolescents aged 10-19 years ( $n$ 3156), National Health and Nutrition Examination Survey 2011-2014

\begin{tabular}{|c|c|c|c|c|c|c|c|}
\hline Rank & Main group & Subgroup & Cons & Mean & SE & PCT & SE \\
\hline 1. & Beverages, Non-alcoholic & Sweetened Beverages & 2087 & $163 \cdot 1$ & $6 \cdot 3$ & $7 \cdot 8$ & 0.3 \\
\hline 2. & Snacks and Sweets & Sweet Bakery Products & 1257 & $144 \cdot 2$ & $7 \cdot 8$ & 6.9 & 0.4 \\
\hline 3. & Mixed Dishes & Mixed Dishes - Pizza & 705 & $138 \cdot 7$ & 11.5 & $6 \cdot 6$ & 0.5 \\
\hline 4. & Grains & Breads, Rolls, Tortillas & 1659 & $122 \cdot 2$ & 4.3 & $5 \cdot 8$ & 0.2 \\
\hline 5. & Mixed Dishes & Mixed Dishes - Grain-based & 715 & $110 \cdot 2$ & $5 \cdot 8$ & $5 \cdot 3$ & 0.3 \\
\hline 6. & Mixed Dishes & Mixed Dishes - Mexican & 495 & $105 \cdot 9$ & $9 \cdot 6$ & 5.0 & 0.5 \\
\hline 7. & Snacks and Sweets & ry Snacks & 1379 & $97 \cdot 3$ & 5.4 & 4.6 & 0.2 \\
\hline 8. & Mixed Dishes & Dishes - Sandwiches & 553 & $97 \cdot 2$ & 8.9 & 4.6 & 0.4 \\
\hline 9. & Milk and Dairy & Milk & 1433 & $91 \cdot 2$ & 3.9 & 4.4 & 0.2 \\
\hline 10. & Protein Foods & & 957 & 84.9 & $7 \cdot 3$ & 4.0 & 0.3 \\
\hline 11. & Ves & Potatoes & 874 & $7 \cdot 7$ & 4.0 & $3 \cdot 2$ & 0.2 \\
\hline 12. & Grains & Cereals & 879 & $56 \cdot 4$ & 3.4 & $2 \cdot 7$ & 0.2 \\
\hline 13. & Prote & Mea & 625 & 53.6 & $3 \cdot 8$ & $2 \cdot 6$ & 0.2 \\
\hline 14. & Snacks and Sweets & Ot & 608 & $52 \cdot 1$ & $5 \cdot 1$ & 2.5 & 0.2 \\
\hline 15. & Mixed Dishes & $-M / P / F$ & 429 & $50 \cdot 8$ & 4.9 & $2 \cdot 4$ & 0.2 \\
\hline 16. & & $c$ & 475 & 8.9 & 4.7 & $2 \cdot 3$ & 0.2 \\
\hline 17. & Sna & & 914 & $5 \cdot 2$ & 4.3 & $2 \cdot 2$ & 0.2 \\
\hline 18. & Milk & & 1017 & $45 \cdot 0$ & 3.3 & $2 \cdot 1$ & 0.2 \\
\hline 19. & Protein Foods & Meats/Poultry & 874 & $42 \cdot 2$ & 3.7 & $2 \cdot 0$ & 0.2 \\
\hline 20. & Fruit & & 1251 & $42 \cdot 0$ & $2 \cdot 4$ & $2 \cdot 0$ & 0.1 \\
\hline 21. & & & 1074 & 1.9 & $3 \cdot 2$ & $2 \cdot 0$ & 0.2 \\
\hline 22. & & Protein Foods & 520 & $40 \cdot 7$ & $3 . \overline{6}$ & 1.9 & 0.2 \\
\hline 23. & Non-alcoholic & & 727 & 34.7 & 3.5 & 1.7 & 0.2 \\
\hline 24. & Mixec & es - Asian & 238 & 33.9 & $5 \cdot 9$ & 1.6 & 0.3 \\
\hline 25. & , Non-alcoholic & & 704 & $32 \cdot 0$ & 4.2 & 1.5 & 0.2 \\
\hline 26. & & & 463 & 0.7 & $2 \cdot 2$ & 1.5 & 0.1 \\
\hline 27. & & & 473 & $30 \cdot 4$ & $2 \cdot 6$ & 1.4 & 0.1 \\
\hline 28. & Milk & & 382 & $27 \cdot 2$ & $2 \cdot 7$ & 1.3 & 0.1 \\
\hline 29. & d Sweets & & 353 & 23.8 & 2.9 & $1 \cdot 1$ & 0.1 \\
\hline 30. & & & 688 & $22 \cdot 6$ & $2 \cdot 9$ & $1 \cdot 1$ & 0.1 \\
\hline 31. & & & 1181 & 20.4 & 1.3 & 1.0 & 0.1 \\
\hline 32. & Mixe & Mix & 337 & $19 \cdot 7$ & $2 \cdot 0$ & 0.9 & 0.1 \\
\hline 33. & Cond & and Sauces & 1282 & $16 \cdot 9$ & 1.3 & 0.8 & 0.1 \\
\hline 34. & Snacks and Sweets & & 181 & $15 \cdot 2$ & $2 \cdot 3$ & 0.7 & 0.1 \\
\hline 35. & Milk and Dairy & Dairy Drinks and Substitutes & 121 & 13.4 & $2 \cdot 1$ & 0.6 & 0.1 \\
\hline 36. & & & 210 & $10 \cdot 9$ & $1 \cdot 8$ & 0.5 & 0.1 \\
\hline 37. & & & 126 & $5 \cdot 7$ & 0.8 & 0.3 & 0.0 \\
\hline 38. & Grair & & 86 & 4.5 & 1.0 & 0.2 & 0.0 \\
\hline 39. & Water & Flavoured or Enhanced Water & 73 & 1.5 & 0.4 & 0.1 & 0.0 \\
\hline 40. & Beverages, Non-alcoholic & Diet Beverages & 234 & 0.7 & $0 \cdot 1$ & 0.0 & 0.0 \\
\hline 41. & Water & Plain Water & 2447 & 0.0 & 0.0 & 0.0 & 0.0 \\
\hline
\end{tabular}

Cons, number of consumers; PCT, percentage contribution; M/P/F, meat/poultry/fish.

*All forty-one weighted subgroups of foods' contribution to daily intake of energy.

†To convert to kJ, multiply kcal values by $4 \cdot 184$. 
burritos and tacos); and $1.7 \%$ from vegetables, excluding potatoes $(0.3 \%$ from other vegetables and combinations). Results are presented in Table 3 and the online supplementary material, Supplemental Table 3.

\section{Contribution of foods to total sodium consumed}

Table 4 and the online supplementary material, Supplemental Table 4, present food subgroups (and categories) contributing to total $\mathrm{Na}$ consumed. The top ten sources contributed $58.0 \%$ of the total and included: $8.7 \%$ from mixed dishes - pizza; $6.7 \%$ from mixed dishes - Mexican (4.6\% from burritos and tacos); $6.6 \%$ from cured meats/ poultry ( $4.7 \%$ from cold cuts and cured meats); $6.5 \%$ from mixed dishes - sandwiches (1.7\% from chicken/turkey sandwiches); $6.3 \%$ from breads, rolls and tortillas $(3.3 \%$ from yeast breads); $6 \cdot 1 \%$ from mixed dishes - grain-based (3.1\% from pasta mixed dishes, excludes macaroni and cheese); $5.5 \%$ from poultry $3.0 \%$ from chicken, whole pieces); $4.1 \%$ from condiments and sauces; $3.9 \%$ from mixed dishes - meat/poultry/fish (2.6\% from meat mixed dishes); and $3.6 \%$ from cheese.

\section{Contribution of foods to total SFA consumed}

The food subgroups (and categories) that contributed to total SFA are presented in Table 5 and the online supplementary material, Supplemental Table 5. The ten highest food sources contributed $64.4 \%$ of total SFA consumed and included: $9.1 \%$ from mixed dishes - pizza; $8.3 \%$ from sweet bakery products (3.4\% from cookies and brownies); $7.9 \%$ from mixed dishes - Mexican (5.2\% from burritos and tacos); $7 \cdot 6 \%$ from milk ( $4.8 \%$ from milk, reduced fat); $6.9 \%$ from cheese; $6.3 \%$ from mixed dishes - sandwiches ( $2.0 \%$ from burgers); $5.4 \%$ from mixed dishes - grain-based ( $2.9 \%$ from macaroni and cheese); $4.8 \%$ from other desserts ( $4.6 \%$ from ice cream and frozen dairy desserts); $4.2 \%$ from fats and oils; and $3.9 \%$ from meats $(2 \cdot 0 \%$ from ground beef).

Table 3 Food sources* of mean and percentage of grams among US adolescents aged 10-19 years ( $n$ 3156), National Health and Nutrition Examination Survey 2011-2014

\begin{tabular}{|c|c|c|c|c|c|c|c|}
\hline Rank & Main group & Subgroup & Cons & Mean & SE & PCT & SE \\
\hline 1. & Water & Plain Water & 2447 & $875 \cdot 6$ & $34 \cdot 8$ & $33 \cdot 1$ & 0.9 \\
\hline 2. & Beverages, Non-alcoholic & Sweetened Beverages & 2087 & 417.6 & $15 \cdot 4$ & $15 \cdot 8$ & 0.6 \\
\hline 3. & Milk and Dairy & Milk & 1433 & $190 \cdot 1$ & 8.6 & $7 \cdot 2$ & 0.3 \\
\hline 4. & Beverages, Non-alcoholic & Coffee and Tea & 704 & 129.5 & 11.5 & 4.9 & 0.4 \\
\hline 5. & Fruit & Fruits & 1251 & $77 \cdot 1$ & 4.9 & $2 \cdot 9$ & 0.2 \\
\hline 6. & Mixed Dishes & Mixed Dishes - Grain-based & 715 & $72 \cdot 0$ & 3.8 & $2 \cdot 7$ & 0.2 \\
\hline 7. & Beverages, Non-alcoholic & $100 \%$ Juice & 727 & $71 \cdot 7$ & $6 \cdot 8$ & $2 \cdot 7$ & 0.2 \\
\hline 8. & Mixed Dishes & Mixed Dishes - Pizza & 705 & 48.9 & 4.0 & 1.9 & 0.2 \\
\hline 9. & Mixed Dishes & Mixed Dishes - Mexican & 495 & $45 \cdot 1$ & 4.2 & 1.7 & 0.2 \\
\hline 10. & Vegetables & Vegetables, excluding Potatoes & 1181 & 44.0 & $2 \cdot 7$ & 1.7 & 0.1 \\
\hline 11. & Grains & Breads, Rolls, Tortillas & 1659 & 43.6 & 1.6 & 1.6 & 0.1 \\
\hline 12. & Beverages, Non-alcoholic & Diet Beverages & 234 & $42 \cdot 8$ & 4.9 & 1.6 & 0.2 \\
\hline 13. & Milk and Dairy & Flavoured Milk & 382 & 38.2 & 3.9 & 1.4 & 0.1 \\
\hline 14. & Mixed Dishes & Mixed Dishes - Sandwiches & 553 & 38.0 & 3.4 & 1.4 & 0.1 \\
\hline 15. & Vegetables & White Potatoes & 874 & 37.8 & $2 \cdot 8$ & 1.4 & 0.1 \\
\hline 16. & oods & & 957 & 35.9 & 3.0 & 1.4 & 0.1 \\
\hline 17. & Mixed Dishes & Dishes - M/P/F & 429 & 35.9 & 3.7 & 1.4 & 0.1 \\
\hline 18. & Snacks and Sweets & Sweet Bakery Products & 1257 & $35 \cdot 3$ & 1.9 & 1.3 & 0.1 \\
\hline 19. & Mixed Dishes & Mixed Dishes - Soups & 337 & 34.0 & 3.3 & 1.3 & 0.1 \\
\hline 20. & and Sweets & rts & 608 & 29.0 & $2 \cdot 8$ & $1 \cdot 1$ & 0.1 \\
\hline 21. & oods & $\mathrm{Me}$ & 625 & 24.5 & $1 \cdot 8$ & 0.9 & 0.1 \\
\hline 22. & Grains & Cool & 463 & 21.9 & 1.7 & 0.8 & 0.1 \\
\hline 23. & Condiments and Sauces & Condiments and Sauces & 1282 & $20 \cdot 7$ & 1.8 & 0.8 & 0.1 \\
\hline 24. & Snacks and Sweets & Savoury Snacks & 1379 & $19 \cdot 9$ & $1 \cdot 1$ & 0.8 & 0.0 \\
\hline 25. & Prot & $\mathrm{Cu}$ & 874 & $19 \cdot 6$ & 1.5 & 0.7 & 0.1 \\
\hline 26. & Mixe & Asian & 238 & $19 \cdot 2$ & $2 \cdot 8$ & 0.7 & 0.1 \\
\hline 27. & Grains & Quick Breads and Bread Products & 475 & $17 \cdot 0$ & 1.5 & 0.6 & 0.1 \\
\hline 28. & Protein Foods & Eggs & 473 & $16 \cdot 6$ & 1.4 & 0.6 & 0.1 \\
\hline 29. & Grains & Ready-to-Eat Cereals & 879 & 14.9 & 0.9 & 0.6 & 0.0 \\
\hline 33. & Milk and & & 1017 & 13.8 & 1.0 & 0.5 & 0.0 \\
\hline 31. & Milk & $\mathrm{D}$ & 121 & 12.6 & $1 \cdot 7$ & 0.5 & 0.1 \\
\hline 32. & Protein Foods & sed Protein Foods & 520 & $12 \cdot 2$ & $1 \cdot 1$ & 0.5 & 0.0 \\
\hline 33. & Water & Flavoured or Enhanced Water & 73 & $10 \cdot 9$ & 1.9 & 0.4 & 0.1 \\
\hline 34. & Fats and Oils & Fats and Oils & 1074 & $10 \cdot 8$ & 0.9 & 0.4 & 0.0 \\
\hline 35. & Snacks and Sweets & & 914 & $10 \cdot 4$ & 1.0 & 0.4 & 0.0 \\
\hline 36. & Sugar & Suge & 688 & 8.1 & $1 \cdot 1$ & 0.3 & 0.0 \\
\hline 37. & Protein Foods & Seafood & 210 & 6.5 & 1.2 & 0.2 & 0.0 \\
\hline 38 & Milk and Dairy & Yoghurt & 126 & $6 \cdot 1$ & 0.9 & 0.2 & 0.0 \\
\hline 39. & Grains & Cooked Cereals & 86 & $5 \cdot 2$ & 1.2 & 0.2 & 0.0 \\
\hline 40. & Snacks and Sweets & Crackers & 353 & $5 \cdot 0$ & 0.6 & 0.2 & 0.0 \\
\hline 41. & Snacks and Sweets & Snack/Meal Bars & 181 & 3.6 & 0.5 & 0.1 & 0.0 \\
\hline
\end{tabular}

Cons, number of consumers; PCT, percentage contribution; M/P/F, meat/poultry/fish.

${ }^{\star}$ All forty-one weighted subgroups of foods' contribution to daily total gram intake. 
Table 4 Food sources* of sodium (mg), mean contribution and as a percentage of total sodium intake, among US adolescents aged 10-19 years ( $n$ 3156), National Health and Nutrition Examination Survey 2011-2014

\begin{tabular}{|c|c|c|c|c|c|c|c|}
\hline Rank & Main group & Subgroup & Cons & Mean & SE & PCT & SE \\
\hline 1. & Mixed Dishes & Mixed Dishes - Pizza & 705 & $300 \cdot 5$ & 25.5 & 8.7 & 0.7 \\
\hline 2. & Mixed Dishes & Mixed Dishes - Mexican & 495 & 231.4 & $21 \cdot 8$ & $6 \cdot 7$ & 0.6 \\
\hline 3. & Protein Foods & Cured Meats/Poultry & 874 & 228.8 & $17 \cdot 0$ & $6 \cdot 6$ & 0.4 \\
\hline 4. & Mixed Dishes & Mixed Dishes - Sandwiches & 553 & $222 \cdot 8$ & 22.5 & 6.5 & 0.6 \\
\hline 5. & Grains & Breads, Rolls, Tortillas & 1659 & $217 \cdot 7$ & $7 \cdot 4$ & $6 \cdot 3$ & 0.2 \\
\hline 6. & Mixed Dishes & Mixed Dishes - Grain-based & 715 & $209 \cdot 1$ & $10 \cdot 0$ & $6 \cdot 1$ & 0.3 \\
\hline 7. & Protein Foods & Poultry & 957 & $189 \cdot 6$ & $16 \cdot 6$ & 5.5 & 0.5 \\
\hline 8. & Condiments and Sauces & Condiments and Sauces & 1282 & 141.9 & 11.4 & $4 \cdot 1$ & 0.3 \\
\hline 9. & Mixed Dishes & Mixed Dishes - M/P/F & 429 & 134.4 & $15 \cdot 6$ & 3.9 & 0.5 \\
\hline 10. & Milk and Dairy & Cheese & 1017 & $124 \cdot 8$ & $10 \cdot 1$ & $3 \cdot 6$ & 0.3 \\
\hline 11. & Snacks and Sweets & ury Snacks & 1379 & $118 \cdot 0$ & 6.9 & 3.4 & 0.2 \\
\hline 12. & $s$ and Sweets & t Bakery Products & 1257 & $113 \cdot 6$ & $6 \cdot 7$ & $3 \cdot 3$ & 0.2 \\
\hline 13. & Vegetables & White Potatoes & 874 & $105 \cdot 8$ & 8.3 & $3 \cdot 1$ & 0.2 \\
\hline 14. & Protein Foods & Meats & 625 & $104 \cdot 2$ & 7.5 & $3 \cdot 0$ & 0.2 \\
\hline 15. & Mixed Dishes & Mixed Dishes - Soups & 337 & $100 \cdot 3$ & $9 \cdot 7$ & $2 \cdot 9$ & 0.3 \\
\hline 16. & Grains & Quick Breads and Bread Products & 475 & 92.4 & 8.6 & $2 \cdot 7$ & 0.2 \\
\hline 17. & Milk & & 1433 & $85 \cdot 8$ & 3.9 & 2.5 & 0.1 \\
\hline 18. & Mixed Dishes & Mixed Dishes - Asian & 238 & $81 \cdot 2$ & 13.8 & $2 \cdot 4$ & 0.4 \\
\hline 19. & Grains & Ready-to-Eat Cereals & 879 & $75 \cdot 0$ & $4 \cdot 3$ & $2 \cdot 2$ & 0.1 \\
\hline 20. & Protein Foods & Eggs & 473 & 73.2 & 6.5 & $2 \cdot 1$ & 0.2 \\
\hline 21. & Fats & $\mathrm{F}$ & 1074 & 68.0 & $6 \cdot 4$ & $2 \cdot 0$ & 0.2 \\
\hline 22. & Non-alcoholic & d Reverar & 2087 & 63.3 & 5.0 & 1.8 & 0.1 \\
\hline 23. & Vegetables & excluding Potatoes & 1181 & 58.8 & 5.4 & 1.7 & 0.2 \\
\hline 24. & Grains & Cooked Grains & 463 & 51.5 & 4.0 & 1.5 & 0.1 \\
\hline 25. & Snacks and Sweets & Crackers & 353 & 41.0 & 5.1 & 1.2 & 0.1 \\
\hline 26. & Prote & ased Protein Foods & 520 & $37 \cdot 2$ & $2 \cdot 7$ & $1 \cdot 1$ & 0.1 \\
\hline 27. & Protein & & 210 & 32.5 & $6 \cdot 7$ & 0.9 & 0.2 \\
\hline 28. & Water & Water & 2447 & $27 \cdot 8$ & 1.3 & 0.8 & 0.0 \\
\hline 29. & Milk and Dairy & red Milk & 382 & $24 \cdot 9$ & $2 \cdot 5$ & 0.7 & 0.1 \\
\hline 30. & Snacks and Sweets & Other Desserts & 608 & $24 \cdot 4$ & $2 \cdot 8$ & 0.7 & 0.1 \\
\hline 31. & Snacks a & & 914 & $14 \cdot 3$ & 1.3 & 0.4 & 0.0 \\
\hline 32. & nd Sweets & Snac & 181 & 11.1 & 1.7 & 0.3 & 0.0 \\
\hline 33. & Milk and Dairy & rinks and Substitutes & 121 & 8.6 & 1.2 & 0.2 & 0.0 \\
\hline 34. & Grains & Cooked Cereals & 86 & $8 \cdot 1$ & $2 \cdot 0$ & 0.2 & 0.1 \\
\hline 35. & Beverages, Non-alcoholic & Coffee and Tea & 704 & 7.4 & 0.5 & 0.2 & 0.0 \\
\hline 36. & Beverages, Non-alcoholic & Diet Beverages & 234 & $5 \cdot 1$ & 0.7 & $0 \cdot 1$ & 0.0 \\
\hline 37. & & Sugars & 688 & 4.7 & 0.6 & 0.1 & 0.0 \\
\hline 38. & Beverages, Non-alcoholic & $100 \%$ Juice & 727 & 3.9 & 0.8 & 0.1 & 0.0 \\
\hline 39. & Milk and Dairy & Yoghurt & 126 & 3.4 & 0.5 & 0.1 & 0.0 \\
\hline 40. & Fruit & Fruits & 1251 & 1.4 & 0.1 & 0.0 & 0.0 \\
\hline 41. & Water & Flavoured or Enhanced Water & 73 & 1.2 & 0.3 & 0.0 & 0.0 \\
\hline
\end{tabular}

Cons, number of consumers; PCT, percentage contribution; M/P/F, meat/poultry/fish.

*All forty-one weighted subgroups of foods' contribution of daily intake of $\mathrm{Na}$.

\section{Contribution of foods to total added sugars consumed}

The added sugar food subgroups (and categories) are presented in Table 6 and the online supplementary material, Supplemental Table 6. The top ten subgroups contributed $89.1 \%$ of total added sugar intake. The top food sources were: $42 \cdot 1 \%$ from SSB $(25 \cdot 6 \%$ from soft drinks); $12 \cdot 1 \%$ from sweet bakery products $(5.0 \%$ from cookies and brownies); $7.6 \%$ from coffee and tea (6.9\% from tea); $6.1 \%$ from candy $(3.5 \%$ from candy not containing chocolate); $5.5 \%$ from other desserts ( $4.3 \%$ from ice cream and frozen dairy desserts); $4.9 \%$ from sugars; $4.9 \%$ from ready-to-eat cereals ( $4.3 \%$ from ready-to-eat cereal, higher in sugar); $2 \cdot 1 \%$ from flavoured milk (0.6\% from flavoured milk, reduced fat); $1.9 \%$ from breads, rolls and tortillas (1.0\% from yeast breads); and $1.8 \%$ from quick breads ( $1 \cdot 1 \%$ from biscuits, muffins, quick breads).

\section{Discussion}

Identification of food sources for intakes of energy, total grams consumed and nutrients to limit may guide public health efforts to align adolescents' diets more closely with current dietary recommendations. The present study provides the food sources of energy, nutrients to limit and total amount of grams consumed by a nationally representative sample of US adolescents; to our knowledge, this is the first time that data for this age group have been reported. While some food categories contributed a high percentage of total energy and total amount of grams consumed, such as milk and water, less desirable food sources, including SSB and sweetened bakery products, were also identified.

The present findings add to the literature that identifies intakes of SSB and sweetened bakery products as being 
Foods commonly consumed by adolescents

Table 5 Food sources* of SFA (g), mean contribution and as a percentage of total SFA intake, among US adolescents aged $10-19$ years $(n 3156)$, National Health and Nutrition Examination Survey 2011-2014

\begin{tabular}{|c|c|c|c|c|c|c|c|}
\hline Rank & Main group & Subgroup & Cons & Mean & SE & РCT & SE \\
\hline 1. & Mixed Dishes & Mixed Dishes - Pizza & 705 & $2 \cdot 4$ & 0.2 & $9 \cdot 1$ & 0.7 \\
\hline 2. & Snacks and Sweets & weet Bakery Products & 1257 & $2 \cdot 2$ & 0.1 & $8 \cdot 3$ & 0.5 \\
\hline 3. & Mixed Dishes & Mixed Dishes - Mexican & 495 & $2 \cdot 1$ & 0.2 & 7.9 & 0.7 \\
\hline 4. & Milk and Dairy & Milk & 1433 & $2 \cdot 0$ & 0.1 & $7 \cdot 6$ & 0.4 \\
\hline 5. & Milk and Dairy & Cheese & 1017 & 1.8 & 0.1 & $6 \cdot 9$ & 0.4 \\
\hline 6. & Mixed Dishes & Mixed Dishes - Sandwiches & 553 & 1.7 & 0.2 & $6 \cdot 3$ & 0.6 \\
\hline 7. & Mixed Dishes & xed Dishes - Grain-based & 715 & 1.4 & 0.1 & 5.4 & 0.4 \\
\hline 8. & Snacks and Sweets & Other Desserts & 608 & 1.3 & 0.1 & 4.8 & 0.5 \\
\hline 9. & Fats and Oils & & 1074 & $1 \cdot 1$ & 0.1 & $4 \cdot 2$ & 0.3 \\
\hline 10. & Protein Foods & & 625 & 1.0 & 0.1 & 3.9 & 0.3 \\
\hline 11. & Foods & & 957 & 1.0 & 0.1 & 3.6 & 0.3 \\
\hline 12. & & Meats/ & 874 & 0.9 & 0.1 & 3.4 & 0.3 \\
\hline 13. & Sweets & & 1379 & 0.9 & 0.1 & 3.4 & 0.3 \\
\hline 14. & Protei & & 473 & 0.7 & 0.1 & $2 \cdot 7$ & 0.2 \\
\hline 15. & Mixed Dishes & d Dishes - M/P/F & 429 & 0.7 & 0.1 & 2.5 & $0 \cdot 2$ \\
\hline 16. & and Sweets & & 914 & 0.7 & 0.1 & 2.5 & 0.2 \\
\hline 17. & & & 874 & 0.6 & 0.1 & $2 \cdot 4$ & 0.2 \\
\hline 18. & & Tortillas & 1659 & 0.5 & 0.0 & 1.9 & 0.1 \\
\hline 19. & n Foods & in Foods & 520 & 0.5 & 0.0 & $1 \cdot 7$ & 0.1 \\
\hline 20. & Grair & Bread Products & 475 & 0.5 & 0.1 & 1.7 & 0.2 \\
\hline 21. & & & 382 & 0.4 & 0.0 & 1.4 & 0.2 \\
\hline 22. & & & 121 & 0.3 & 0.1 & $1 \cdot 2$ & 0.2 \\
\hline 23. & & & 337 & 0.3 & 0.0 & 1.0 & 0.1 \\
\hline 24. & Mixe & s - Asian & 238 & 0.2 & 0.0 & 0.9 & 0.2 \\
\hline 25. & d Sweets & & 353 & 0.2 & 0.0 & 0.8 & 0.1 \\
\hline 26. & auces & & 1282 & 0.2 & 0.0 & 0.7 & 0.1 \\
\hline 27. & & & 879 & 0.2 & 0.0 & 0.7 & 0.1 \\
\hline 28. & & xcluding Potatoes & 1181 & $0 \cdot 1$ & 0.0 & 0.5 & 0.0 \\
\hline 29. & ges, Non-alcoholic & & 704 & $0 \cdot 1$ & 0.0 & 0.4 & 0.1 \\
\hline 30. & and Swee & & 181 & $0 \cdot 1$ & 0.0 & 0.4 & 0.1 \\
\hline 31. & & & 210 & $0 \cdot 1$ & 0.0 & 0.4 & 0.1 \\
\hline 32. & Non-alcoholic & & 2087 & 0.1 & 0.0 & 0.3 & 0.1 \\
\hline 33. & & $\mathrm{C}$ & 463 & $0 \cdot 1$ & 0.0 & 0.2 & 0.0 \\
\hline 34. & Milk and Dairy & & 126 & $0 \cdot 1$ & 0.0 & $0 \cdot 2$ & 0.0 \\
\hline 35. & Sugars & & 688 & $0 \cdot 1$ & 0.0 & 0.2 & 0.1 \\
\hline 36. & & Cooked Cereals & 86 & 0.0 & 0.0 & 0.2 & 0.0 \\
\hline 37. & Fruit & & 1251 & 0.0 & 0.0 & $0 \cdot 1$ & 0.0 \\
\hline 38. & Bever & & 727 & 0.0 & 0.0 & 0.0 & 0.0 \\
\hline 39. & Beverages, Non-alcoholic & Diet Beverages & 234 & 0.0 & 0.0 & 0.0 & 0.0 \\
\hline 40. & Water & & 2447 & 0.0 & 0.0 & 0.0 & 0.0 \\
\hline 41. & Water & Flavoured or Enhanced Water & 73 & 0.0 & 0.0 & 0.0 & 0.0 \\
\hline
\end{tabular}

Cons, number of consumers; $\mathrm{PCT}$, percentage contribution; M/P/F, meat/poultry/fish.

*All forty-one weighted subgroups of foods' contribution of daily intake of SFA.

major contributors of added sugars and SFA. However, the question that has not been addressed in the research field is whether there is an independent role of nutrients to limit and food sources, or if nutrients to limit and food sources simply add excessive energy to the diet leading to adverse health effects ${ }^{(36)}$. For example, $100 \%$ fruit juice contributes positively to nutrient intakes, but should be consumed in adequate portion sizes in order not to overconsume excess energy. On the other hand, flavoured teas and coffees contribute to higher amount of added sugars. A previous study ${ }^{(36)}$ suggests that the role of SSB is probably larger than has been reported because the SSB category did not include sugar-sweetened tea and coffee.

Corroborating with a representative study using 20092012 data from NHANES ${ }^{(36)}$ showed that the major food source of added sugars was sweet bakery products (i.e. cookies and brownies). The total intake of added sugars was $12 \%$ in the adolescent population. Moreover, sweetened bakery products contributed to the intake of SFA in the diet of the US population ${ }^{(37)}$. Therefore, practitioners, researchers and policy makers should continuously be working together to reduce intakes of added sugars and SFA from the food sources commonly consumed by adolescents, in accordance with the recommendations of the Dietary Guidelines for Americans ${ }^{(5)}$.

In the current study, adolescents reported consuming an average of $8774 \mathrm{~kJ}(2097 \mathrm{kcal})^{(5)}$, which is within current estimated daily energy needs ${ }^{(38)}$. However, approximately 37 and $20 \%$ of adolescents were overweight or obese, respectively ${ }^{(39)}$. By inference, many must be consuming more energy than they require. Moreover, studies have repeatedly shown under-reporting of energy intake based on self-reported data, specifically among overweight and obese individuals ${ }^{(40-42)}$. 
Table 6 Food sources* of added sugar (teaspoons), mean contribution and as a percentage of total added sugar intake, among US adolescents aged $10-19$ years ( $n 3156)$, National Health and Nutrition Examination Survey 2011-2014

\begin{tabular}{|c|c|c|c|c|c|c|c|}
\hline Rank & Main group & Subgroup & Cons & Mean & SE & PCT & SE \\
\hline 1. & Beverages, Non-alcoholic & Sweetened Beverages & 2087 & 8.6 & 0.3 & $42 \cdot 1$ & $1 \cdot 3$ \\
\hline 2. & Snacks and Sweets & Sweet Bakery Proc & 1257 & 2.5 & 0.2 & $12 \cdot 1$ & 0.7 \\
\hline 3. & Beverages, Non-alcoholic & Coffee and Tea & 704 & 1.6 & 0.2 & $7 \cdot 6$ & $1 \cdot 1$ \\
\hline 4. & Snacks and Sweets & Candy & 914 & 1.3 & $0 \cdot 1$ & $6 \cdot 1$ & 0.6 \\
\hline 5. & Snacks and Sweets & Other Desserts & 608 & $1 \cdot 1$ & 0.1 & 5.5 & 0.5 \\
\hline 6. & Sugars & Sugars & 688 & 1.0 & 0.1 & 4.9 & 0.6 \\
\hline 7. & Grains & Ready-to-Eat Cereals & 879 & 1.0 & 0.1 & 4.9 & 0.4 \\
\hline 8. & Milk and Dairy & ed Milk & 382 & 0.4 & 0.0 & $2 \cdot 1$ & 0.2 \\
\hline 9. & Grains & Breads, Rolls, Tortillas & 1659 & 0.4 & 0.0 & 1.9 & 0.1 \\
\hline 10. & Grains & Quick Breads and Bread Products & 475 & 0.4 & 0.0 & 1.8 & 0.2 \\
\hline 11. & Condiments and Sauces & s and Sauces & 1282 & 0.3 & 0.0 & 1.2 & 0.2 \\
\hline 12. & Snac & $\mathrm{Sn}$ & 181 & 0.2 & 0.0 & $1 \cdot 1$ & 0.1 \\
\hline 13. & Mixed & - Sandwiches & 553 & 0.2 & 0.0 & $1 \cdot 1$ & 0.1 \\
\hline 14. & Milk and Dairy & Dairy Drinks and Substit & 121 & 0.2 & 0.0 & $1 \cdot 1$ & 0.2 \\
\hline 15. & Mixed Dishes & Mixed Dishes - Asian & 238 & 0.1 & 0.0 & 0.7 & 0.2 \\
\hline 16. & Fruit & & 1251 & 0.1 & 0.0 & 0.6 & 0.1 \\
\hline 17. & Fat & & 1074 & 0.1 & 0.0 & 0.6 & 0.1 \\
\hline 18. & Milk & & 126 & 0.1 & 0.0 & 0.6 & 0.1 \\
\hline 19. & Mixed Dishes & Dishes - Pizza & 705 & 0.1 & 0.0 & 0.5 & 0.1 \\
\hline 20. & Mixed Dishes & $s-M / P / F$ & 429 & 0.1 & 0.0 & 0.5 & 0.2 \\
\hline 21. & Water & & 73 & 0.1 & 0.0 & 0.5 & 0.1 \\
\hline 22. & Mix & & 715 & 0.1 & 0.0 & 0.4 & 0.1 \\
\hline 23. & ad Sweets & & 353 & 0.1 & 0.0 & 0.3 & 0.0 \\
\hline 24. & and Sweets & Snacks & 1379 & 0.0 & 0.0 & 0.2 & $0 \cdot 1$ \\
\hline 25. & Foods & ed Protein Foods & 520 & 0.0 & 0.0 & 0.2 & 0.0 \\
\hline 26. & & & 874 & 0.0 & 0.0 & 0.2 & 0.0 \\
\hline 27. & Prot & & 957 & 0.0 & 0.0 & 0.2 & 0.0 \\
\hline 28. & Mixe & & 495 & 0.0 & 0.0 & 0.1 & 0.0 \\
\hline 29. & Vegetables & excluding Potatoes & 1181 & 0.0 & 0.0 & 0.1 & 0.0 \\
\hline 30. & Vegetables & & 874 & 0.0 & 0.0 & 0.1 & 0.0 \\
\hline 31. & & & 86 & 0.0 & 0.0 & 0.0 & 0.0 \\
\hline 32. & s, Non-alcoholic & Diet & 234 & 0.0 & 0.0 & 0.0 & 0.0 \\
\hline 33. & Prote & $\mathrm{Me}$ & 625 & 0.0 & 0.0 & 0.0 & 0.0 \\
\hline 34. & Protein Foods & Seafood & 210 & 0.0 & 0.0 & 0.0 & 0.0 \\
\hline 35. & Mixed Dishes & Mixed Dishes - Soups & 337 & 0.0 & 0.0 & 0.0 & 0.0 \\
\hline 36. & Beverages, Non-alcoholic & & 727 & 0.0 & 0.0 & 0.0 & 0.0 \\
\hline 37. & & & 463 & 0.0 & 0.0 & 0.0 & 0.0 \\
\hline 38. & & & 1017 & 0.0 & 0.0 & 0.0 & 0.0 \\
\hline 39. & Protein Foods & Eggs & 473 & 0.0 & 0.0 & 0.0 & 0.0 \\
\hline 40. & Milk and Dairy & Milk & 1433 & 0.0 & 0.0 & 0.0 & 0.0 \\
\hline 41. & Water & Plain Water & 2447 & 0.0 & 0.0 & 0.0 & 0.0 \\
\hline
\end{tabular}

Cons, number of consumers; PCT, percentage contribution; M/P/F, meat/poultry/fish.

*All forty-one weighted subgroups of foods' contribution of daily intake of added sugar.

Although previous studies with NHANES data did not evaluate adolescents specifically, consistent findings were found for children aged 6-18 years $^{(12)}$ and $\geq 2$ years $^{(14,36,43)}$ in that the highest-ranking food sources of Na were pizza, mixed dishes - Mexican, mixed dishes sandwiches, cheese, milk and poultry. For SFA, major food sources were beef, milk and cheese; and for added sugars major food sources were desserts, snacks, and SSB. Meeting nutrient intake recommendations while staying within energy needs has proven to be challenging for many adolescents ${ }^{(12,14,17)}$, potentially contributing to the high prevalence of overweight and obesity ${ }^{(39)}$, premature risk for CVD and type 2 diabetes $^{(44)}$ and a high likelihood of remaining with these adverse health conditions into adult life ${ }^{(45)}$. Average $\mathrm{Na}$ intake among US adolescents is much higher than in younger children and adults ${ }^{(32)}$. Corroborating with a previous NHANES analysis ${ }^{(12)}$ the top food sources of $\mathrm{Na}$ were mixed dishes (i.e. pizza and Mexican), cured meats, bread rolls and tortilla, and savoury snacks, but with variation in the ranking of the top food sources' contributions by different social demographic characteristics. A previous study ${ }^{(46)}$ of consumption trends showed that individuals aged 4-19 years significantly reduced their consumption of solid fats, added sugars and $\mathrm{Na}$ in the last decade because grocery stores, fast-food restaurants and full-service restaurants have been making an effort to reduce those nutrients to limit. To help US adolescents improve dietary habits and reduce intakes of $\mathrm{Na}$, SFA and added sugars with better food choices, identifying food sources contributing to energy, nutrients to limit and total gram amount consumed is essential and needs continued monitoring.

Strengths of the present study are the use of a large, nationally representative database to examine food 
sources of selected nutrients. The limitations include selfreported measurements that may over- or under-report dietary intake and physical activity ${ }^{(31)}$. Results need to be interpreted with caution because adolescents might be over-reporting their intakes of healthier foods and underreporting their intakes of food sources of nutrients to limit in the diet, particularly when using a one $24 \mathrm{~h}$ dietary recall $^{(47)}$. Further, under-reporting is greater in those who are overweight or obese compared with those in the healthy weight range ${ }^{(42,48)}$ and findings might need to be interpreted with caution. To reduce the impact of misreporting, data were expressed as percentage contributions of food sources to energy, grams and nutrients to limit ${ }^{(49)}$. A single day's intake may not be representative of an individual's usual intake but given large sample sizes does adequately represent mean intakes. Also, the identification of food sources of nutrient intakes is a limitation because this depends on a food grouping system that may not be universally used. For example, other evidence using the NOVA classification system ${ }^{(49)}$ and the Food Pyramid Groups ${ }^{(50)}$ have identified some foods that were in the top intake sources of adolescents, while in the present analysis those food sources were not among the top major contributors. Finally, the study used cross-sectional data from which casual relationships cannot be drawn.

\section{Conclusion}

Given adolescence is an important period of transition from childhood to adulthood with increased freedom of food choices, understanding dietary intake during this period is important ${ }^{(50)}$. The present study provides detailed information on the food sources contributing to total energy, total grams, Na, SFA and added sugars consumed by a representative sample of US adolescents aged 10-19 years. A large proportion of total energy consumed was from energy-dense food groups (e.g. SSB and sweet bakery products), but also from some major food sources contributing important vitamins and minerals (e.g. beef, cheese and milk) ${ }^{(14)}$. Awareness of food sources may be useful to create or refine dietary strategies within public health initiatives to improve the diets of adolescents.

\section{Acknowledgements}

Financial support: This research received no specific grant from any funding agency in the public, commercial or notfor-profit sectors. This work is a publication of the USDA/ ARS Children's Nutrition Research Center, Department of Pediatrics, Baylor College of Medicine, Houston, TX, USA. The contents of this publication do not necessarily reflect reviews or policies of the USDA, nor does mention of trade names, commercial products or organizations imply endorsement from the USDA. Conflict of interest: As Vice
President of Nutrition Impact, LLC, V.F. performs data analyses of large government databases like NHANES for numerous members of the food, beverage and dietary supplement industry. T.N. has received numerous grants from the food and beverage industry and federal agencies throughout her career. Authorship: All authors contributed to the development of this study. A.C.L. contributed to interpretation of data, drafting the manuscript and critical revision of the manuscript for scientific content. T.B. contributed to critical revision of the manuscript for scientific content. D.T. contributed to critical revision of the manuscript for scientific content. S.T. contributed to critical revision of the manuscript for scientific content. C.O'.N. contributed to critical revision of the manuscript for scientific content. V.F contributed to statistical analysis and critical revision of the manuscript for scientific content. T.N., the senior researcher of this project, participated in study concept and design, acquisition of data, interpretation of data and critical revision of the manuscript for important scientific content. Ethics of buman subject participation: As it was a secondary analysis which lacked personal identifiers, this study did not require institutional review beyond the approval from the Centers for Disease Control and Prevention, National Center for Health Statistics Research Ethics Review Board (https://www.cdc.gov/nchs/nhanes/irba98.htm, accessed 6 April 2018).

\section{Supplementary material}

To view supplementary material for this article, please visit https://doi.org/10.1017/S1368980018002884

\section{References}

1. Keast DR, Fulgoni VL 3rd, Nicklas TA et al. (2013) Food sources of energy and nutrients among children in the United States: National Health and Nutrition Examination Survey 2003-2006. Nutrients 5, 283-301.

2. Taber DR, Chriqui JF, Perna FM et al. (2012) Weight status among adolescents in States that govern competitive food nutrition content. Pediatrics 130, 437-444.

3. Previdelli AN, de Andrade SC, Fisberg RM et al. (2016) Using two different approaches to assess dietary patterns: hypothesis-driven and data-driven analysis. Nutrients $\mathbf{8}$, E593.

4. Thorpe MG, Kestin M, Riddell LJ et al. (2014) Diet quality in young adults and its association with food-related behaviours. Public Health Nutr 17, 1767-1775.

5. Dietary Guidelines Advisory Committee (2015) Scientific Report of the 2015 Dietary Guidelines Advisory Committee: Advisory Report to the Secretary of Health and Human Services and the Secretary of Agriculture. https://health. gov/dietaryguidelines/2015-scientific-report/PDFs/ScientificReport-of-the-2015-Dietary-Guidelines-Advisory-Committee. pdf (accessed January 2018).

6. Utley JM, Affuso O \& Rucks AC (2016) Adolescent obesity in contextual settings: a scoping study of multilevel and hierarchical examinations. Clin Obes 6, 296-304. 
7. Taber DR, Chriqui JF, Vuillaume R et al. (2015) The association between state bans on soda only and adolescent substitution with other sugar-sweetened beverages: a crosssectional study. Int J Behav Nutr Phys Act 12, Suppl. 1, S7.

8. Deierlein AL, Galvez MP, Yen IH et al. (2014) Local food environments are associated with girls' energy, sugarsweetened beverage and snack-food intakes. Public Health Nutr 17, 2194-2200.

9. Vedovato GM, Trude AC, Kharmats AY et al. (2015) Degree of food processing of household acquisition patterns in a Brazilian urban area is related to food buying preferences and perceived food environment. Appetite 87, 296-302.

10. Slining MM, Mathias KC \& Popkin BM (2013) Trends in food and beverage sources among US children and adolescents: 1989-2010. J Acad Nutr Diet 113, 1683-1694.

11. Rodriguez LA, Madsen KA, Cotterman C et al. (2016) Added sugar intake and metabolic syndrome in US adolescents: crosssectional analysis of the National Health and Nutrition Examination Survey 2005-2012. Public Health Nutr 19, 2424-2434.

12. Quader ZS, Gillespie C, Sliwa SA et al. (2017) Sodium intake among US school-aged children: National Health and Nutrition Examination Survey, 2011-2012. J Acad Nutr Diet 117, 39-47.e5.

13. Slining MM \& Popkin BM (2013) Trends in intakes and sources of solid fats and added sugars among US children and adolescents: 1994-2010. Pediatr Obes 8, 307-324.

14. Huth PJ, Fulgoni VL, Keast DR et al. (2013) Major food sources of calories, added sugars, and saturated fat and their contribution to essential nutrient intakes in the US diet: data from the National Health and Nutrition Examination Survey (2003-2006). Nutr J 12, 116.

15. US Department of Health and Human Services \& US Department of Agriculture (2015) 2015-2020 Dietary Guidelines for Americans, 8th ed. https://health.gov/diet aryguidelines/2015/guidelines/ (accessed January 2018).

16. Dunford EK, Poti JM \& Popkin BM (2017) Emerging disparities in dietary sodium intake from snacking in the US population. Nutrients 9, E610.

17. O'Neil CE, Keast DR, Fulgoni VL et al. (2012) Food sources of energy and nutrients among adults in the US: NHANES 2003-2006. Nutrients 4, 2097-2120.

18. Centers for Disease Control and Prevention, National Center for Health Statistics (2013) NHANES I Web Tutorial I Survey Design Factors I Survey Design. https://www.cdc.gov/nchs/ tutorials/NHANES/SurveyDesign/SampleDesign/intro_i.htm (accessed January 2018).

19. Zipf G, Chiappa M, Porter KS et al. (2013) National health and nutrition examination survey: plan and operations, 1999-2010. Vital Health Stat 1 issue 56, 1-37.

20. Centers for Disease Control and Prevention, National Center for Health Statistics (updated 2017) National Health and Nutrition Examination Survey | Questionnaires, Datasets, and Related Documentation I NHANES Response Rates and Population Totals. https://wwwn.cdc.gov/nchs/nhanes/ ResponseRates.aspx (accessed January 2018).

21. Ahluwalia N, Dwyer J, Terry A et al. (2016) Update on NHANES dietary data: focus on collection, release, analytical considerations, and uses to inform public policy. Adv Nutr 7, 121-134.

22. Centers for Disease Control and Prevention, National Center for Health Statistics (2017) National Health and Nutrition Examination Survey I Survey Participants I Welcome NHANES Participants I What does participation involve? https://www.cdc.gov/nchs/ nhanes/participant-eligibility.htm (accessed January 2018).

23. Anon. (2003) Age limits and adolescents. Paediatr Child Health 8, 577-578.

24. Centers for Disease Control and Prevention (2013) Unweighted Response Rates for NHANES 2011-2012 by Age and Gender. https://www.cdc.gov/nchs/data/nhanes/ response_rates_cps/rrt1112 (accessed January 2018).
25. Centers for Disease Control and Prevention (2015) Unweighted Response Rates for NHANES 2013-2014 by Age and Gender. https://www.cdc.gov/nchs/data/nhanes/ response_rates_cps/2013_2014_response_rates (accessed January 2018).

26. Centers for Disease Control and Prevention, National Center for Health Statistics (2015) National Health and Nutrition Examination Survey I 2013-2014 Data Documentation, Codebook, and Frequencies I Demographic Variables and Sample Weights (DEMO_H) I Data File: DEMO_H.xpt. https://wwwn.cdc.gov/Nchs/Nhanes/2013-2014/DEMO_H. htm. (accessed January 2018).

27. Centers for Disease Control and Prevention, National Center for Health Statistics (2015) National Health and Nutrition Examination Survey I 2013-2014 Data Documentation, Codebook, and Frequencies I Physical Activity (PAQ_H) | Data File: PAQ_H.xpt. https://wwwn.cdc.gov/Nchs/ Nhanes/2013-2014/PAQ_H.htm (accessed January 2018).

28. Blanton CA, Moshfegh AJ, Baer DJ et al. (2006) The USDA Automated Multiple-Pass Method accurately estimates group total energy and nutrient intake. J Nutr 136, 2594-2599.

29. US Department of Agriculture, Agricultural Research Service, Beltsville Human Nutrition Research Center (updated 2018) Overview of Food Patterns Equivalents Database. https://www.ars.usda.gov/northeast-area/ beltsville-md-bhnrc/beltsville-human-nutrition-researchcenter/food-surveys-research-group/docs/fped-overview/ (accessed May 2018).

30. National Health and Nutrition Examination Survey (2002) MEC In-Person Dietary Interviewers Procedures Manual. https://www.cdc.gov/nchs/data/nhanes/nhanes_03_04/ dietary_mec.pdf (accessed January 2018).

31. Naska A, Lagiou A \& Lagiou P (2017) Dietary assessment methods in epidemiological research: current state of the art and future prospects. F1000Res $\mathbf{6}, 926$.

32. US Department of Agriculture, Agricultural Research Service, Beltsville Human Nutrition Research Center (updated 2018) What We Eat in America data tables. Nutrient intakes: from foods/beverage. https://www.ars.usda.gov/northeastarea/beltsville-md-bhnrc/beltsville-human-nutrition-researchcenter/food-surveys-research-group/docs/wweianhanesoverview/ (accessed May 2018).

33. Centers for Disease Control and Prevention (2007) National Health and Nutrition Examination Survey (NHANES): Anthropometry Procedures Manual, https://www.cdc.gov/ nchs/data/nhanes/nhanes_07_08/manual_an.pdf (accessed January 2018).

34. National Institutes of Health, National Heart, Lung, and Blood Institute (2002) Third Report of the National Cholesterol Education Program (NCEP) Expert Panel on Detection, Evaluation and Treatment of High Blood Cholesterol in Adults (Adult Treatment Panel III): Final Report. https://www.nhlbi.nih.gov/files/docs/resources/heart/atp-3cholesterol-full-report.pdf (accessed January 2018).

35. Centers for Disease Control and Prevention, National Centers for Health Statistics (2016) CDC Growth Charts. https://www.cdc.gov/growthcharts/cdc_charts.htm (accessed January 2018).

36. Bailey RL, Fulgoni VL III, Cowan AE et al. (2018) Sources of added sugars in young children, adolescents, and adults with low and high intakes of added sugars. Nutrients $\mathbf{1 0}$, 102.

37. Papanikolaou Y \& Fulgoni VL (2017) Certain grain foods can be meaningful contributors to nutrient density in the diets of US children and adolescents: data from the National Health and Nutrition Examination Survey, 2009-2012. Nutrients 9, E160. 
38. Institute of Medicine (2002) Dietary Reference Intakes for Energy, Carbohydrate, Fiber, Fat, Fatty Acids, Cholesterol, Protein, and Amino Acids. Washington, DC: The National Academies Press.

39. Skinner AC, Perrin EM \& Skelton JA (2016) Prevalence of obesity and severe obesity in US children, 1999-2014. Obesity (Silver Spring). 24, 1116-1123.

40. Rangan A, Allman-Farinelli M, Donohoe E et al. (2014) Misreporting of energy intake in the 2007 Australian Children's Survey: differences in the reporting of food types between plausible, under- and over-reporters of energy intake. J Hum Nutr Diet 27, 450-458.

41. Avelino GF, Previdelli AN, Castro MA et al. (2014) Underreporting of energy intake and associated factors in a population-based study. Cad Saude Publica 30, 663-668.

42. Scagliusi FB, Ferriolli E, Pfrimer K et al. (2008) Underreporting of energy intake in Brazilian women varies according to dietary assessment: a cross-sectional study using doubly labeled water. J Am Diet Assoc 108, 2031-2040.

43. Welsh JA, Sharma AJ, Grellinger L et al. (2011) Consumption of added sugars is decreasing in the United States. Am J Clin Nutr 94, 726-734.

44. O'Connor EA, Evans CV, Burda BU et al. (2017) Screening for obesity and intervention for weight management in children and adolescents: evidence report and systematic review for the US Preventive Services Task Force. JAMA 317, 2427-2444.

45. Celermajer DS \& Ayer JG (2006) Childhood risk factors for adult cardiovascular disease and primary prevention in childhood. Heart 92, 1701-1706.

46. Rehm CD \& Drewnowski A (2016) Trends in consumption of solid fats, added sugars, sodium, sugar-sweetened beverages, and fruit from fast food restaurants and by fast food restaurant type among US children, 2003-2010. Nutrients 8, E804.

47. Collins CE, Watson J \& Burrows T (2010) Measuring dietary intake in children and adolescents in the context of overweight and obesity. Int J Obes (Lond) 34, 1103-1115.

48. Rangan AM, Flood VM \& Gill TP (2011) Misreporting of energy intake in the 2007 Australian Children's Survey: identification, characteristics and impact of misreporters. Nutrients 3, 186-199.

49. Collins CE, Dewar DL, Schumacher TL et al. (2013) 12-Month changes in dietary intake of adolescent girls attending schools in low-income communities following the NEAT Girls cluster randomized controlled trial. Appetite 73, 147-155.

50. Lipsky LM, Nansel TR, Haynie DL et al. (2017) Diet quality of US adolescents during the transition to adulthood: changes and predictors. Am J Clin Nutr 105, 1424-1432. 